\title{
PERSEU - Ferramenta Computacional para a Prevenção do DORT Causado pelo uso Contínuo do Computador
}

\author{
Lucas Albertins de Lima', Ana Esther V. Barbosa', Waldemar P. Ferreira Neto ${ }^{3}$, \\ Joseana Macêdo Fechine ${ }^{4}$ \\ 1,2,3 Integrante do Grupo PET-Computação \\ Curso de Graduação em Ciência da Computação \\ ${ }^{4}$ Tutora do Grupo PET Computação \\ Departamento de Sistemas e Computação \\ Universidade Federal de Campina Grande \\ 58.109-970, Campina Grande, PB, Brasil \\ \{lucas, aesther, neto, joseana\}@dsc.ufcg.edu.br
}

\begin{abstract}
Resumo. O PERSEU é uma ferramenta computacional que visa auxiliar no combate aos DORT/LER (Distúrbios Osteomusculares Relacionados com Trabalho/Lesões por Esforços Repetitivos) provenientes do uso contínuo do computador. O PERSEU busca promover uma melhor interação entre o homem e computador, pois os problemas relacionados aos Distúrbios Osteomusculares têm se tornado cada vez mais comuns entre os usuários de computador, chegando até aos casos de paralisia dos membros e articulações do usuário. Portanto, de uma forma simples e eficaz, a ferramenta propõe a realização de pausas e exercícios durante a atividade computacional, tornando assim esta interação menos danosa ao corpo humano.
\end{abstract}

\section{Introdução}

O software PERSEU busca conjugar a Tecnologia da Informação e a Fisioterapia no combate a um mal cada vez mais comum no mundo moderno, especialmente entre usuários de computador, mal esse denominado DORT/LER - Distúrbios Osteomusculares Relacionados ao Trabalho/Lesões por Esforços Repetitivos (CODO, 1995). Embora não seja um problema recente, os DORT/LER vêm assumindo um caráter preocupante.

O projeto PERSEU tomou como base a existência de uma correlação entre o uso do computador de maneira contínua e excessiva, e o surgimento de distúrbios osteomusculares. Deste modo, o software PERSEU visa investigar o nível de morbidade osteomuscular em usuários de computador que se enquadram no perfil de sujeitas aos DORT. Para tanto, o software será aplicado em acadêmicos do Curso de Ciência da Computação de uma Instituição de Ensino Superior, mas especificamente nos acadêmicos da Universidade Federal de Campina Grande (UFCG). 
O PERSEU é uma ferramenta computacional destinada a auxiliar na prevenção e tratamento de lesões por esforços repetitivos e, para isso, a ferramenta deverá administrar o uso do computador e auxiliar a utilizá-lo da melhor forma, através do acompanhamento do ritmo de trabalho do usuário. A ferramenta fornece exercícios diversos, de modo a estimular a pausa e a realização de um exercício de alongamento por parte do usuário durante um curto intervalo de tempo, de forma a prevenir as lesões causadas por atividades repetitivas.

O software terá licença de software livre e código aberto e ficará disponível via Internet para os usuários preocupados com a sua saúde e para desenvolvedores que pretendam aperfeiçoá-lo ou estudá-lo.

As seções seguintes deste artigo estão organizadas da seguinte forma: na Seção 2 são apresentados conceitos importantes dos DORT. Em seguida, na Seção 3 é apresentada uma breve descrição dos trabalhos relevantes no contexto do PERSEU, sendo realizada uma análise comparativa dos modelos existentes. Na Seção 4, o software PERSEU é descrito com detalhes. Por fim, na Seção 5 são apresentadas as considerações finais.

\section{Princípios dos DORT}

Os DORT tornaram-se a mais nova epidemia dos últimos anos, já que a partir da década de 80 passaram a ser causa freqüente de afastamento do trabalho (CODO, 1995).

As condições de trabalho, que podem levar ao aparecimento de sintomas dos DORTS, incluem movimentos repetitivos, aplicação de forças (principalmente com as mãos) levantamento e transporte de pesos, posturas inadequadas e estresse, relacionado às condições psicossociais em que o trabalho acontece. O aparecimento das afecções está ligado à exposição dos trabalhadores a esses riscos e sua magnitude depende da intensidade, freqüência e duração da exposição e da capacidade individual de lidar com as demandas do trabalho (MACIEL, 2000).

Dentre as principais regiões do corpo que podem ser acometidas por DORT, a coluna vertebral, especialmente a região lombar e cervical têm sido nos últimos anos as mais afetadas, seguidas de entorses de tornozelo e joelho. A postura sentada durante muitas horas, no uso do computador, provoca a situação de vulnerabilidade e de risco de se adquirir DORT (VIEIRA, 2000).

As causas do DORT estão relacionadas ao trabalho realizado, sendo assim para prevenir o surgimento desse mal é necessário modificar as condições de trabalho que podem potencialmente ocasionar este mal.

\section{Trabalhos Relacionados}

Existem hoje no mercado várias ferramentas relacionadas à prevenção dos DORT em usuários de computador. Porém, a maioria das ferramentas disponíveis não é escrita para a língua portuguesa, o que dificulta o uso por parte de diversos usuários que não dominam as línguas com quais as ferramentas são desenvolvidas. Além disso, a maior parte dessas ferramentas não é gratuita.

As principais ferramentas pesquisadas foram as seguintes: RSIGuard Versão 4.0 (RSIGUARD, 2005), Rest Bitz Versão 2.10 (RESTBITZ, 2000), ErgoEnterprise Single 
(ERGO, 2006), Stress Away Versão Demo (STRESS, 2006) e ErgoSense Versão Demo 2.0.2 (ERGOSENSE, 1999).

No Quadro 1 é apresentada uma análise comparativa entre o PERSEU e as ferramentas pesquisadas, considerando os aspectos mais relevantes relacionados ao foco de interesse do PERSEU.

Quadro 1 Comparação entre o Perseu e as ferramentas pesquisadas.

\begin{tabular}{|c|c|c|c|c|c|c|}
\hline $\begin{array}{c}\text { Funcionalidades/ } \\
\text { Ferramentas }\end{array}$ & PERSEU & $\begin{array}{c}\text { RSIGuard } \\
\text { Versão 3.0 }\end{array}$ & $\begin{array}{c}\text { Rest } \\
\text { Bitz } \\
\text { Versão } \\
2.10\end{array}$ & $\begin{array}{c}\text { ErgoEnterprise } \\
\text { Single }\end{array}$ & $\begin{array}{c}\text { Stress } \\
\text { Away } \\
\text { Versão } \\
\text { Demo }\end{array}$ & $\begin{array}{c}\text { ErgoSen } \\
\text { se } \\
\text { Versão } \\
\text { Demo } \\
2.0 .2\end{array}$ \\
\hline $\begin{array}{c}\text { Língua } \\
\text { Portuguesa }\end{array}$ & Sim & Não & Não & Não & Não & Não \\
\hline Uso de Vídeos & Sim & Sim & Não & Sim & Sim & Sim \\
\hline Configurável & Não & Sim & Sim & Sim & Sim & Sim \\
\hline $\begin{array}{c}\text { Ajuda (on-line ou } \\
\text { na ferramenta) }\end{array}$ & Não & Sim & Sim & Sim & Sim & Sim \\
\hline Código aberto & Sim & Não & Não & Não & Não & Não \\
\hline Freeware & Sim & Sim & Sim & Não & Não & Não \\
\hline
\end{tabular}

\section{Construção do Software PERSEU}

Para a construção do PERSEU, tornou-se necessário definir quais os aspectos relevantes na elaboração de uma ferramenta computacional com o objetivo de auxiliar na prevenção/tratamento dos DORT. Para tanto, foi realizada uma parceria com um grupo de pesquisa do Curso de Fisioterapia da Universidade Estadual da Paraíba (UEPB) ${ }^{1}$, coordenado por uma especialista na área, a professora Vitória Regina Quirino de Araújo.

Inicialmente, o grupo de Fisioterapia, em parceria com a equipe de desenvolvimento do projeto, elaborou um questionário para verificar o nível de incidência dos DORT no público alvo (docentes e discentes do Departamento de Sistemas e Computação da Universidade Federal de Campina Grande, DSC/UFCG). Em seguida, esse questionário foi aplicado com o objetivo de dispor dos dados necessários para a análise da viabilidade de construção do software. Nas seções a seguir serão descritos os processos de levantamento dos dados (a partir do questionário) e de construção do software.

\subsection{Levantamento dos Dados}

O modelo do questionário desenvolvido foi baseado no Nordic Musculoskeletal Questionnaire (NMQ), Questionário Nórdico de Sintomas (QNSO), (DICKINSON, 1992). Esse questionário foi aplicado em dois grupos: corpo discente e corpo docente do DSC/UFCG. Para tanto, foi utilizada a Amostragem Aleatória Estratificada, com uma amostra composta de 109 e 23 respondentes, para cada grupo, respectivamente, o que

\footnotetext{
${ }^{1}$ O grupo de pesquisa do Curso de Fisioterapia da UEPB é coordenado pela Professora Vitória Regina Quirino e formado por mais duas alunas: Priscilla Dias de Souza e Aline Christine de Souza Silva.
}

Anais do III Simpósio Brasileiro de Sistemas de Informação. Curitiba, PR, outubro de 2006. 
corresponde a uma amostra representativa da população em estudo, em torno de $30 \%$ e $60 \%$, respectivamente.

Os resultados obtidos comprovaram a apresentação de fortes sintomas de distúrbios osteomusculares em praticamente todos os grupos musculares da comunidade estudada, independente do sexo, tanto para os discentes quanto os docentes, conforme Tabelas 1 e 2. Os respondentes podem apresentar mais de um distúrbio osteomuscular. Assim sendo, a soma dos percentuais ultrapassa $100 \%$.

Tabela 1 Distribuição dos distúrbios osteomusculares segundo a região do corpo acometida (comunidade docente).

\begin{tabular}{c|c}
\hline Local & \% da Amostra \\
\hline Pescoço e Ombros & 77,77 \\
\hline Membros Superiores & 22,22 \\
\hline Alto das Costas & 33,33 \\
\hline Baixo das Costas & 66,67 \\
\hline
\end{tabular}

Tabela 2 Distribuição de distúrbios osteomusculares segundo a região do corpo acometida (comunidade discente).

\begin{tabular}{c|c}
\hline Local & \% da Amostra \\
\hline Pescoço e Ombros & 60,00 \\
\hline Membros Superiores & 48,60 \\
\hline Alto das Costas & 50,00 \\
\hline Baixo das Costas & 51,40 \\
\hline
\end{tabular}

Após o levantamento dos dados e a análise dos resultados, por parte do especialista, foram realizadas reuniões para definir as características do software a ser desenvolvido. Foram identificados os seguintes requisitos para o software:

1. O software deverá ser executado em background, de forma a não interferir nas demais atividades que estarão sendo realizadas pelo usuário;

2. A interação com o usuário deverá ser realizada na língua portuguesa;

3. O software deverá sugerir os exercícios, não devendo exigir do usuário a tomada de decisões, quanto à escolha do exercício que preferir realizar;

4. O software deverá possibilitar a realização de interrupções periódicas a partir do uso do teclado e do mouse;

5. A sugestão dos exercícios deverá ser realizada na forma textual e visual (vídeos), para se tornar mais didático e atrativo para o usuário;

6. A ferramenta deverá dispor de mais de um exercício para cada tipo de necessidade, o que tornará a ferramenta mais ilustrativa e mais estimulante para o usuário.

\subsection{Software PERSEU}


Para a produção do PERSEU, foram observadas as características de outras ferramentas, conforme apresentado na Seção 3 e os requisitos observados na seção anterior.

De posse dessas informações, partiu-se para a definição da linguagem de programação a ser utilizada. Dentre Java, C++ e Delphi, a última foi escolhida por atender às necessidades de possibilitar a execução em background e poder captar de forma simples todas as interações vindas do teclado e mouse, por ser uma linguagem compilada que permite gerar arquivos executáveis que podem trabalhar no nível de sistema operacional. Vale salientar que estas características também estão disponíveis na linguagem $\mathrm{C}++$, mas o que levou a utilização de Delphi foi a experiência de alguns membros da equipe com esta linguagem.

Após a definição dos requisitos, foi elaborado o Modelo de Navegação para a ferramenta (Figuras 1 e 2), segundo MORI (2003). Na Figura 1 é detalhado o comportamento do software, desde a sua inicialização até a fase de exibição dos exercícios. Na Figura 2 é possível observar o comportamento da ferramenta no tocante à exibição das informações relevantes (descrição e vídeos ilustrativos) para a execução do exercício sugerido.

A metodologia adotada para a construção da ferramenta tomou como base três componentes: A Lógica da Aplicação, Sugestão de Exercícios e Exibição de Exercícios.

O componente da Lógica da Aplicação realiza o processamento relevante para o monitoramento das ações do usuário. Após verificar a necessidade de realização de um exercício, este componente se comunica com o componente de Sugestão de Exercícios, que indica um exercício ao usuário, depois de uma carga de uso do computador. Se o usuário aceitar a sugestão, será dado início ao componente de Exibição de Exercícios, sendo exibida uma tela para o usuário, contendo um vídeo e um texto correspondente de um determinado exercício para um grupo muscular, sugerido pelo programa.

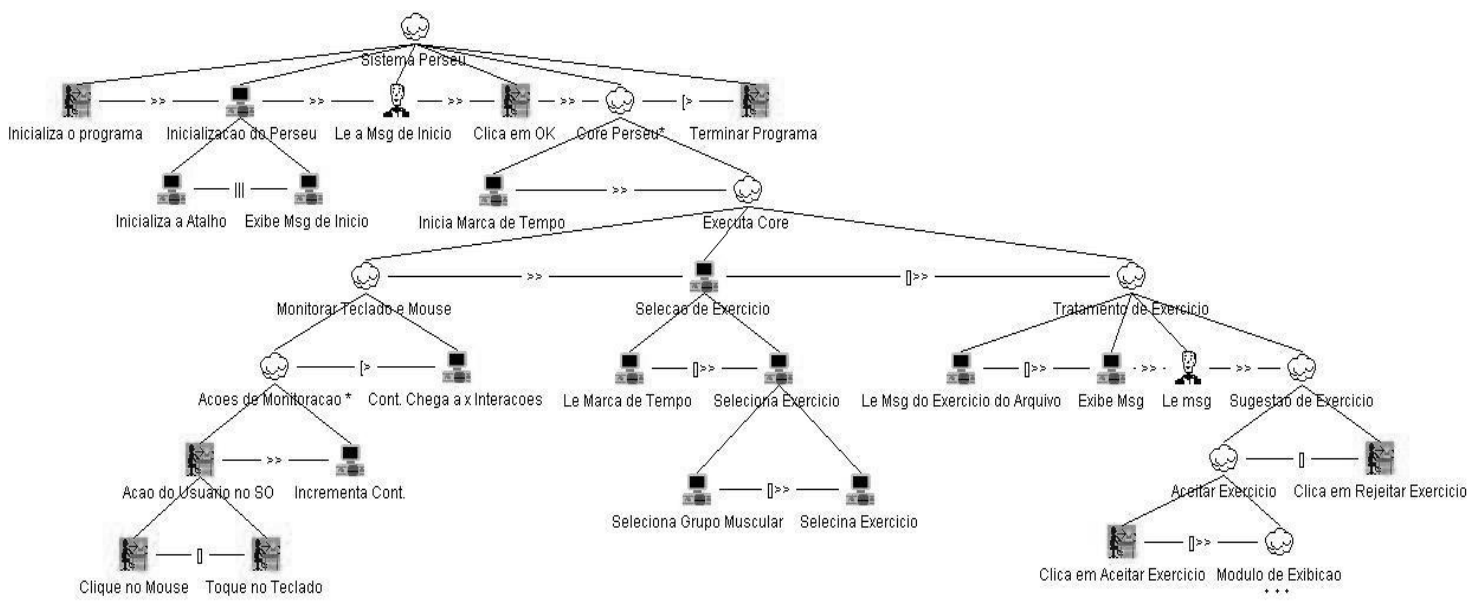

Figura 1 Modelo de Navegação do PERSEU. 


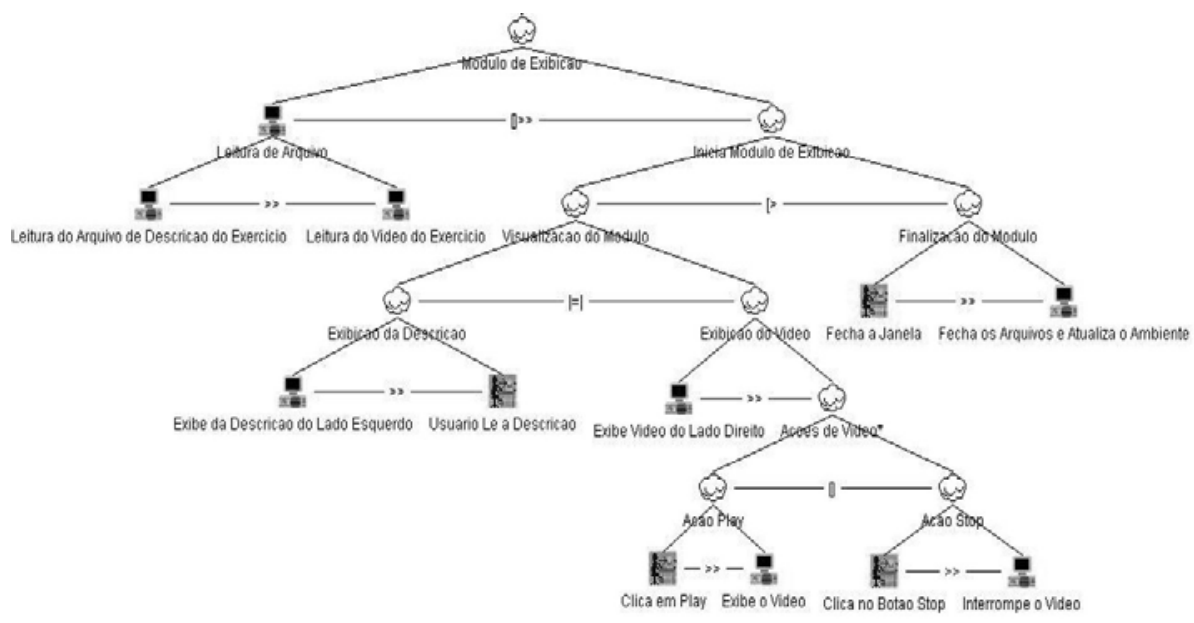

Figura 2 Destaque para o Modelo de Navegação do Módulo de Exibição.

Em seguida, ao usuário fechará a tela exibida, o componente da Lógica da Aplicação reinicia o monitoramento, formando assim um ciclo entre os três componentes. Este último ciclo se mantém até que a ferramenta seja finalizada. O diagrama de seqüência, apresentado na Figura 3, ilustra a forma de comunicação entre os componentes da ferramenta.

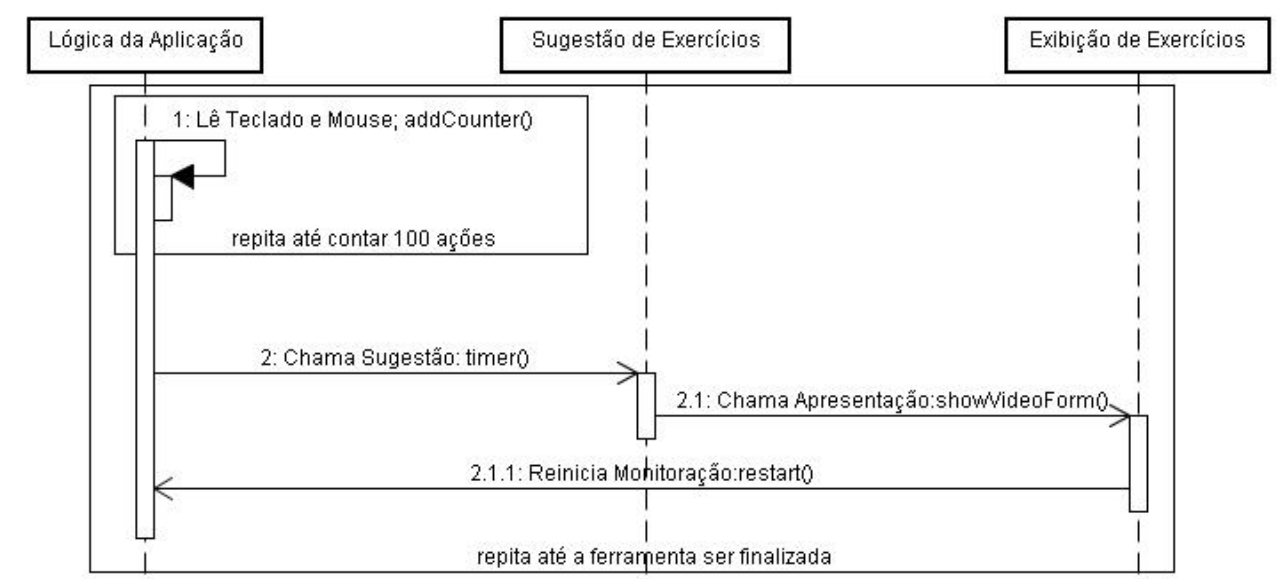

Figura 3 Diagrama de Seqüência dos componentes da Lógica da Aplicação, Sugestão de Exercícios e Exibição de Exercícios do PERSEU.

\subsubsection{Componente da Lógica de Aplicação}

Este componente executa em um form invisível (em background), transparente para o usuário. Ele disponibiliza um ícone na barra de ferramentas do sistema operacional, que possibilita ao usuário habilitar ou desabilitar o programa.

A lógica funciona da seguinte forma: a cada 10 milissegundos é lido o estado do teclado e dos botões do mouse, caso esse status esteja ativado, incrementa-se o contador de ações do usuário. Após determinado número de ações do usuário, é analisado o tempo decorrido entre as sugestões de exercícios e o contador de ações. A partir dessa análise, o programa pode disparar um evento, que apresenta a sugestão de exercício para o usuário. Depois de detectada a necessidade de realização de um evento de sugestão de exercício, o software seleciona o grupo muscular que deverá ser exercitado. 
Em seguida, o componente da lógica invoca o próximo componente, o de Sugestão de Exercícios.

Como o objetivo da ferramenta é realizar a contagem do número de eventos do mouse e/ou do teclado, esta não monitora a(s) tarefa(s) que está(ão) sendo realizada(s) pelo usuário, de forma a garantir a sua privacidade.

\subsubsection{Componente de Sugestão de Exercícios}

A principal função desse componente é exibir na tela uma mensagem alertando o usuário sobre sua situação em relação ao uso computador, sugerindo um exercício. A mensagem a ser exibida é lida de um arquivo com o nome do exercício proposto.

Na tela exibida, o usuário terá duas opções a escolher realizar ou não o exercício. Se usuário não desejar realizar o exercício, deverá clicar no botão <cancelar> ou fechar janela, e o componente da Lógica da Aplicação reiniciará o monitoramento do uso do computador pelo usuário. Porém, se o usuário clicar no botão <continuar>, o componente de Exibição de Exercícios será iniciado e, ao término da execução desse componente, o componente da Lógica de Aplicação também recomeçará a monitorar a freqüência de pressionamento de teclas ou cliques de mouse realizados pelo usuário.

\subsubsection{Componente de Exibição de Exercícios}

A principal função desse módulo é exibir uma série de vídeos e informação textual ensinando ao usuário como realizar o exercício ergonômico. Para cada tipo de atividade realizada pelo usuário, será disponibilizado um exercício, definido pela especialista. Por exemplo, se o usuário utilizou o mouse durante determinado intervalo de tempo, isto poderá provocar bursite no ombro por uso excessivo do braço e do ombro em elevação quando o mouse está distante do corpo na mesa de trabalho. Dessa forma, será sugerido um exercício (ou conjunto de exercícios) visando minimizar este problema.

Neste componente o software abrirá um novo form, no qual é apresentada uma descrição do exercício que o usuário deverá desempenhar na parte esquerda, e um componente de vídeo na parte direita (Figura 4). O usuário poderá iniciar, pausar, voltar e avançar o vídeo. Serão disponibilizados vários vídeos para cada exercício, tornando o uso da ferramenta mais interessante.

Ao final da exibição do vídeo, o usuário poderá fechar a janela. Em seguida, o componente da Lógica da Aplicação voltará a realizar suas tarefas, de forma transparente, entrando em um novo ciclo até que o usuário desabilite a ferramenta.

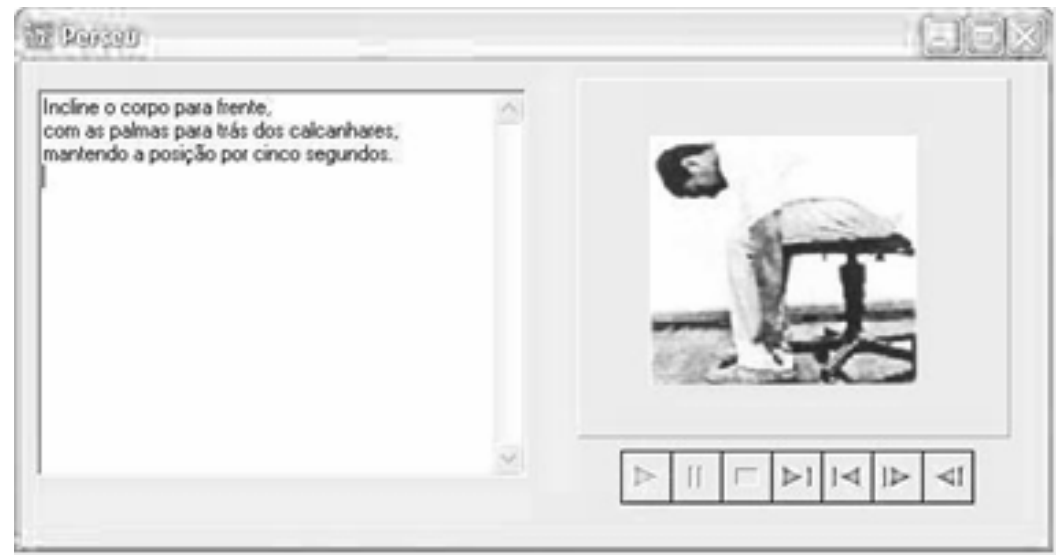

Anais do III Simpósio Brasileiro de Sistemas de Informação. Curitiba, PR, outubro de 2006. 
Figura 4 Interface exposta pelo Componente de Exibição de Exercícios.

\section{Considerações Finais}

O levantamento quantitativo apresentado no artigo mostra coerência com as indicações da especialista a respeito do DORT. A combinação de fatores, como excesso de horas trabalhadas, ausência de pausas e ritmo acelerado, provoca o surgimento de DORT.

Os usuários de computador não atribuem devida atenção e importância ao risco de DORT. Muitos só tomam atitudes de prevenção/tratamento após apresentar o problema. A única arma para lidar com o problema é a prevenção. Nesse sentido, o PERSEU é uma ferramenta computacional que busca a melhoria das condições de uso do computador, fortalecendo a interação entre a informática, a sociedade e o indivíduo.

O software está em fase de conclusão, dependendo ainda de alguns requisitos de interface e da produção dos vídeos. Os vídeos estão sendo desenvolvidos por uma equipe do curso de Arte e Mídia da UFCG e tão logo sejam produzidos, serão incorporados à ferramenta. Já existe um protótipo da ferramenta com as principais funcionalidades implementadas, o qual já foi apresentado e aprovado pelo especialista.

Vale ressaltar, que após a sua conclusão, a ferramenta será disponibilizada na WEB, junto com o seu código fonte, para o seu uso e aperfeiçoamento, seguindo assim as concepções de código aberto e software livre.

\section{Referências}

CODO, W., ALMEIDA, M. C. de (1995) “LER - Lesões por Esforços Repetitivos”, In: L.E.R. Lesões por Esforços Repetitivos, Editora Vozes. $4^{\mathrm{a}}$ edição.

DICKINSON C. E., CAMPION K., FOSTER A., NEWMAN S. (1992) “Questionnaire development: an examination of the Nordic Musculoskeletal Questionnaire”, Applied Ergonomics.

ERGO (2006) “ErgoEnterprise Single”, http://www.windowsmarketplace.com/ details.aspx?view=info\&itemId=2075124, junho.

ERGOSENSE (1999) “ErgoSense Versão Demo 2.0.2”, http://www.omniquad.com/omniquad_ergosense.htm, junho.

MACIEL, R. H. (2000) "Prevenção da LER/DORT - O que a Ergonomia pode oferecer”, http://www.cnbcut.com.br/social/ts12.htm, agosto.

RESTBITZ (2000) "Restbitz ${ }^{\mathrm{TM}} \quad$ V2.10: Formerly BreakTime", http://www.littlehummingbird.com/software/restbitz/, junho.

RSIGUARD (2005), "RSIGuard The most powerful ergonomic software available for the management of Repetitive Strain Injuries”, http://www.rsiguard.com, maio.

STRESS (2006) "Stress Away Software Versão Demo”, http://www.stressaway.com, junho.

VIEIRA, V. L. M. (2000) "Prevenção das LER/DORT em pessoas que trabalham sentados e usuários do computador”, http://www.pclq.usp.br/jornal/prevencao.htm, setembro. 
MORI, G., PATERNÒ, F., SANTORO, C (2003) "Tool Support for Designing Nomadic Applications Proceedings of IUI 2003”, Miami, Florida. 